\title{
Simplified Chain Inflation
}

\author{
Qing-Guo Huang \\ School of physics, Korea Institute for Advanced Study, \\ 20\%-43, Cheongryangri-Dong, Dongdaemun-Gu, \\ Seoul 130-722, Korea \\ huangqg@kias.re.kr
}

\begin{abstract}
We propose a simplified chain inflation model and calculate the primordial power spectra of the scalar and tensor fluctuations. The spectral index and the tensor-scalar ratio are respectively 0.972 and 0.089 which are consistent with present cosmological observations.
\end{abstract}


Inflation models proposed by Guth in [1] not only explain the large-scale homogeneity and isotropy of the universe, but also provide a natural mechanism to generate the observed magnitude of inhomogeneity (see $[2,3]$ etc. for a review). During the period of inflation, quantum fluctuations are generated within the Hubble horizon, which then stretch outside the horizon to become classical. In the subsequent deceleration phase after inflation these frozen fluctuations re-enter the horizon, and seed the matter and radiation density fluctuations observed in the universe.

In the last two decades many inflation models were proposed. However, none of them can be naturally realized in a fundamental theory. Gravity governs the background evolution during inflation and quantum effects provide a natural mechanism to generate the primordial perturbations to seed the fluctuations for forming the large scale structure of the universe and the anisotropies in cosmic microwave background radiation which can be measured precisely. A quantum theory of gravity is needed before we understand the full theory in the early universe. Nowadays string theory, as we know, is the only self-consistent quantum theory of gravity. String theory can only live in ten-dimensional spacetime. We need to compact ten-dimensional string theory to four dimensions. Many free parameters appear after compactifications, even though there is no free dimensionless parameter in string theory in ten dimensions. Recent developments for the flux compactifications $[4,5]$ suggest that a huge number of meta-stable string vacua emerge in string theory. The whole space of such string vacua is called string landscape.

Cosmological observations implies that the density perturbation is roughly $\delta \rho / \rho \sim$ $10^{-5}$ in our universe. A flat potential of the inflaton is called for in inflation model. On the other hand, a flat potential naturally brings a large enough number of e-folds during inflation to solve the problems of hot big bang model. Usually we can expect that one or more small dimensionless parameters in the potential of inflaton is related to the small density perturbation. On question we should ask is why there is such a small parameter in a fundamental theory. Since the number of the meta-stable vacua in string landscape is so huge, it offers an opportunity to explain this small number. Actually many inflation models in string theory, such as [6-10] and so on, have been proposed in the last few years. But what is the distinguishing phenomenon for string landscape is still an open question. The authors in [11] suggested that the string landscape has a model independent prediction which says that our universe should be spatially open.

Even though the shape of string landscape has not been figured out, heuristically we expect that the meta-stable vacuum with large vacuum energy has a very short life time. In [12], Tye suggested that our universe would decay rapidly from a site with large vacuum energy to a long-lived meta-stable vacuum with a small positive cosmological constant 
through the resonance tunneling. Thus it is possible to dynamically solve today's dark energy problem. However, inflation is still needed in the early universe. Fortunately, chain inflation [13-15] becomes generic in this scenario. In this model, the universe tunneled rapidly through a series of meta-stable vacua with different vacuum energies. After many tunneling events, more than 60 e-folds are obtained and the problems in hot big bang are solved. In [16], the authors use numerical method to calculate the amplitude of the primordial power spectra. However, their simulating results implies a quite large tensorscalar ratio which is not consistent with cosmological observations. 1

In this short note, we propose a simplified chain model and suggest a new method to compute the density perturbations, spectral index and tensor-scalar ratio. Our results perform a nice fit to the data.

In chain inflation model, the universe begins in a meta-stable vacuum with a large positive vacuum energy. The system could tunnel to the lower minima along a variety of possible directions in the string landscape. However, many of the neighboring minima is inaccessible, since the tunneling probabilities into them are too small. In $[18,19]$, the authors investigated a classical field theory in which there are two homogeneous equilibrium states with different energy densities. In the quantum version of the theory, the state of higher energy density becomes unstable through barrier penetration. To be simple, we only quantified the tunneling probability by modeling a single tunneling event for a scalar field theory with potential

$$
V(\phi)=\frac{1}{4} \lambda\left(\phi^{2}-a^{2}\right)^{2}+\frac{\sigma}{2 a}(\phi-a),
$$

where $\sigma$ is the vacuum energy difference between two neighboring meta-stable vacua. The tunneling probability is roughly given by

$$
\Gamma \sim \exp \left(-\frac{27 \pi^{2}}{2} \frac{\kappa^{4}}{\sigma^{3}}\right)
$$

where $\kappa$ is the tension of the brane interpolating between the two vacua. In [20,21], Guth et al. showed that the probability of a point remaining in a false de Sitter vacuum is roughly given by

$$
p(t) \sim e^{-\frac{4 \pi}{3} \beta H t},
$$

here the dimensionless parameter $\beta$ is

$$
\beta=\frac{\Gamma}{H^{4}},
$$

\footnotetext{
${ }^{1}$ In [16], the amplitude of the scalar power spectrum is given by $\Delta_{\mathcal{R}}^{2} \sim \beta / \alpha^{2}$. On the other hand, the power spectrum for the tensor modes is roughly the same as that for the perturbation of the scalar field. The amplitude of the tensor perturbations in [16] is $\Delta_{T}^{2} \sim \beta$. Thus the tensor-scalar ratio takes the form $r=\Delta_{T}^{2} / \Delta_{\mathcal{R}}^{2} \sim \alpha^{2}$. However, their simulating results shows that $\alpha$ is much larger than 1 , which contradicts to WMAP3 [17] where $r \leq 0.65$ (95\% CL).
} 
and $H$ is the Hubble parameter in this vacuum. Thus the lifetime of the field in this meta-stable vacuum is estimated as

$$
\tau \simeq \frac{3}{4 \pi H \beta}
$$

A lower bound on the dimensionless parameter $\beta$ is obtained

$$
\beta \geq \frac{9}{4 \pi}
$$

in order for percolation and thermalization to be achieved. In this paper, we focus on the case with the lifetime of the meta-stable vacua much short than Hubble time $H^{-1}$, or equivalently $\beta \gg \frac{3}{4 \pi}$.

In the simplified chain inflation we assume $\sigma$ is always a constant. Since inflation responsible for the observed modes of the density perturbations only lasts a few e-folds, the Hubble parameter $H$ can be taken as a constant during this period. Naively the vacuum energy goes like

$$
\rho_{V} \simeq \rho_{V, i}-\frac{\sigma}{\tau} t
$$

where $\rho_{V, i}$ is the initial vacuum energy. The vacuum energy drops $\sigma /(H \tau)$ per Hubble time $H^{-1}$. Here we also assume $\sigma$ is much smaller than $\rho_{V}$ and then the series of tunneling events can be regarded as a continuous evolution of a scalar field. The Hubble parameter is governed by Friedmann equation

$$
H^{2}=\frac{\rho_{V}}{3 M_{p}^{2}}=\frac{\rho_{V, i}-\frac{\sigma}{\tau} t}{3 M_{p}^{2}},
$$

where $M_{p}$ is the reduced Planck scale. The number of e-folds before the end of inflation is related to the energy density $\rho_{V}$ by

$$
N_{e}=\int_{t}^{t_{\text {end }}} H\left(t^{\prime}\right) d t^{\prime} \simeq \frac{2}{3 \sqrt{3}} \frac{\tau}{M_{p} \sigma} \rho_{V}^{3 / 2}
$$

or equivalently,

$$
\begin{aligned}
\rho_{V} & \simeq\left(\frac{3 \sqrt{3}}{2} \frac{M_{p} \sigma}{\tau} N_{e}\right)^{\frac{2}{3}}, \\
H^{2} & \simeq\left(\frac{\sigma / \tau}{2 M_{p}^{2}} N_{e}\right)^{\frac{2}{3}} .
\end{aligned}
$$

According to eq. (8), the "slow-roll" parameter $\epsilon$ is given by

$$
\epsilon \equiv-\frac{\dot{H}}{H^{2}}=\frac{\sigma / \tau}{6 M_{p}^{2} H^{3}}=\frac{1}{3 N_{e}} .
$$


The meaning of $\sigma / \tau$ is nothing but the change of the vacuum energy per time unit. Generically the tunneling from one site to another site with a lower cosmological constant is accompanied by some radiation due to the bubble percolation, nucleation and collision. During inflation radiation is inflated away. Suppose the change of the vacuum energy at the last step is large, which implies that the change of the vacuum energy per time unit is quite large and then the "slow-roll" condition $\epsilon \ll 1$ is broken down. On the other hand, the loss of the vacuum energy is transferred to radiation and then the energy density is dominated by radiation. Now chain inflation ended and reheating happened.

Now the amplitude of the primordial power spectra for the scalar and tensor perturbations can be expressed as respectively

$$
\begin{aligned}
& \Delta_{\mathcal{R}}^{2}=\frac{H^{2} / M_{p}^{2}}{8 \pi^{2} \epsilon}=\frac{3}{2^{11 / 3} \pi^{2}}\left(\frac{\sigma / \tau}{M_{p}^{5}}\right)^{\frac{2}{3}} N_{e}^{\frac{5}{3}}, \\
& \Delta_{T}^{2}=\frac{H^{2} / M_{p}^{2}}{\pi^{2} / 2}=\frac{2^{1 / 3}}{\pi^{2}}\left(\frac{\sigma / \tau}{M_{p}^{5}}\right)^{\frac{2}{3}} N_{e}^{\frac{2}{3}} .
\end{aligned}
$$

The spectral index and the tensor-scalar ratio are given by

$$
\begin{aligned}
n_{s} & =1+\frac{d \ln \Delta_{\mathcal{R}}^{2}}{d \ln k} \simeq 1-\frac{d \ln \Delta_{\mathcal{R}}^{2}}{d N_{e}}=1-\frac{5}{3 N_{e}}, \\
r & =\frac{\Delta_{T}^{2}}{\Delta_{\mathcal{R}}^{2}}=\frac{16}{3 N_{e}} .
\end{aligned}
$$

For $N_{e}=60$, the spectral index and the tensor ratio are respectively $n_{s}=0.972$ and $r=0.089$. The results of WMAP three-year data are presented in [17]. To properly compare our results with WMAP results, we show our results in Fig. 1. Our results are nicely compatible with WMAP at $95 \%$ confidence level.

Using the WMAP normalization $\Delta_{\mathcal{R}}^{2}=19.9_{-1.8}^{+1.3} \times 10^{-10}$, we find

$$
\frac{\sigma / \tau}{M_{p}^{5}}=8.6 \times 10^{-16} .
$$

The Hubble parameter and the energy density at $N_{e}=60$ are respectively $H \simeq 3 \times 10^{-5} M_{p}$ and $\rho_{V} \simeq 2.7 \times 10^{-9} M_{p}^{4}$. Requiring $\sigma \ll \rho_{V}$ yields

$$
\frac{\tau}{t_{p}} \ll 3 \times 10^{6}, \quad \text { or } \quad \frac{\tau}{H^{-1}} \ll 90,
$$

where $t_{p}=M_{p}^{-1}$ is the Planck time. Our requirement that the lifetime of the meta-stable vacua is much shorter than Hubble time $H^{-1}$ is reasonable.

To summarize, a simplified chain inflation model is proposed. In this model, the vacuum energy drops only a little per step and the series of tunneling just looks like the 

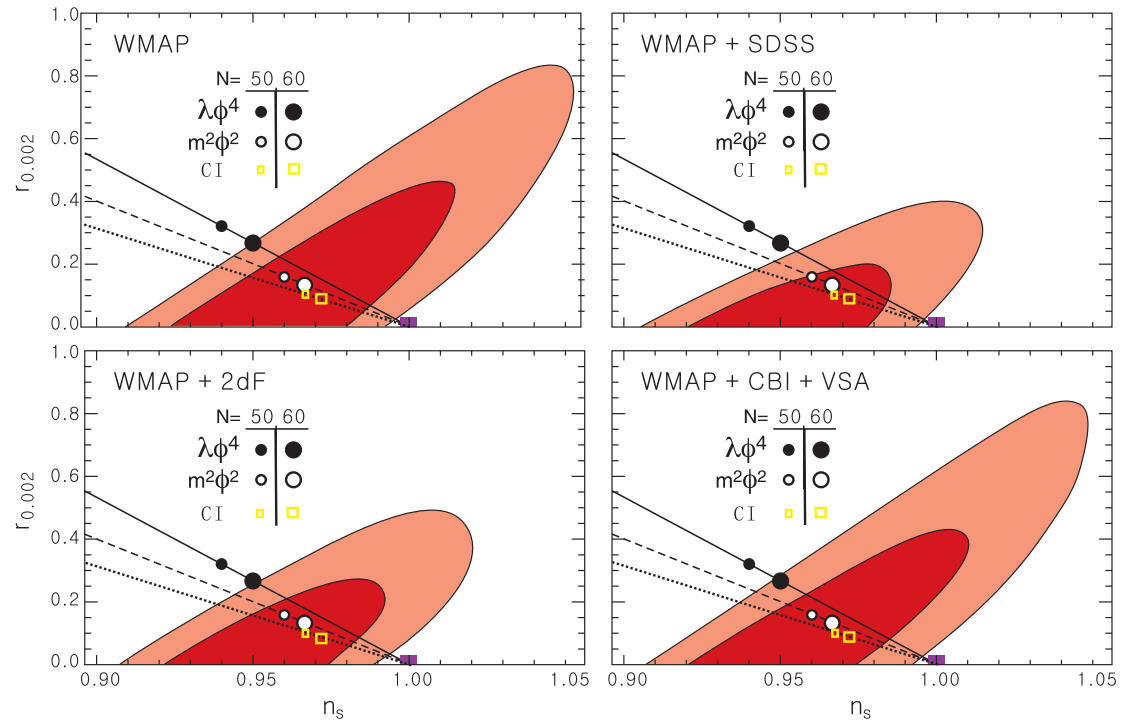

Figure 1: "CI" denotes chain inflation.

continuous evolution. We also estimate the density perturbations and we find our model can fit the present data very well.

In the simplified chain inflation, we assume the vacuum energy drops the same energy density $\sigma$ in each tunneling event. However we do not expect it really happens in string landscape where the situation becomes much more complicated. It is even possible that in some steps along the chain the system tunnels to the vacua with slightly higher energy density. But we can take our semi-quantitative calculation as an averaged effect. Our results only depend on $\sigma / \tau$. Naively we suggest that $\sigma / \tau$ should be replaced by the average value $\langle\sigma / \tau\rangle$ in string landscape. Generically there are vast number of chains, but only a few of them can satisfy what we have observed. The amplitude of the scalar power spectra depends on the detail of the chain. But the spectral index and the tensor-scalar ratio can be taken as the quite insensitive predictions of string landscape.

\section{Acknowledgments}

We would like to thank $\mathrm{H}$. Tye for useful comments. We also acknowledge the use of one figure from WMAP collaboration. 


\section{References}

[1] A. H. Guth, "The Inflationary Universe: A Possible Solution To The Horizon And Flatness Problems," Phys. Rev. D 23, 347 (1981).

[2] V. F. Mukhanov, H. A. Feldman and R. H. Brandenberger, "Theory of cosmological perturbations. Part 1. Classical perturbations. Part 2. Quantum theory of perturbations. Part 3. Extensions," Phys. Rept. 215, 203 (1992).

[3] D. H. Lyth and A. Riotto, "Particle physics models of inflation and the cosmological density perturbation," Phys. Rept. 314, 1 (1999) arXiv:hep-ph/9807278.

[4] S. B. Giddings, S. Kachru and J. Polchinski, "Hierarchies from fluxes in string compactifications," Phys. Rev. D 66, 106006 (2002) arXiv:hep-th/0105097].

[5] S. Kachru, R. Kallosh, A. Linde and S. P. Trivedi, "De Sitter vacua in string theory," Phys. Rev. D 68, 046005 (2003) arXiv:hep-th/0301240].

[6] S. Kachru, R. Kallosh, A. Linde, J. M. Maldacena, L. McAllister and S. P. Trivedi, "Towards inflation in string theory," JCAP 0310, 013 (2003) arXiv:hep-th/0308055.

[7] J. J. Blanco-Pillado et al., "Racetrack inflation," JHEP 0411, 063 (2004) arXiv:hep-th/0406230.

[8] J. J. Blanco-Pillado et al., "Inflating in a better racetrack," JHEP 0609, 002 (2006) arXiv:hep-th/0603129.

[9] R. Allahverdi, A. R. Frey and A. Mazumdar, "Graceful exit from a stringy landscape via MSSM inflation," arXiv:hep-th/0701233.

[10] K. i. Maeda and N. Ohta, "Inflation from M-theory with fourth-order corrections and large extra dimensions," Phys. Lett. B 597, 400 (2004) arXiv:hep-th/0405205];

K. i. Maeda and N. Ohta, "Inflation from superstring / M theory compactification with higher order corrections. I," Phys. Rev. D 71, 063520 (2005) arXiv:hep-th/0411093;

K. Akune, K. i. Maeda and N. Ohta, "Inflation from superstring / M-theory compactification with higher order corrections. II: Case of quartic Weyl terms," Phys. Rev. D 73, 103506 (2006) arXiv:hep-th/0602242;

N. Ohta, "Accelerating cosmologies and inflation from M / superstring theories," Int. J. Mod. Phys. A 20, 1 (2005) arXiv:hep-th/0411230.

[11] B. Freivogel, M. Kleban, M. Rodriguez Martinez and L. Susskind, "Observational consequences of a landscape," JHEP 0603, 039 (2006) arXiv:hep-th/0505232]. 
[12] S. H. Henry Tye, "A new view of the cosmic landscape," arXiv:hep-th/0611148.

[13] K. Freese and D. Spolyar, "Chain inflation: 'Bubble bubble toil and trouble'," JCAP 0507, 007 (2005) arXiv:hep-ph/0412145.

[14] K. Freese, J. T. Liu and D. Spolyar, "Inflating with the QCD axion," Phys. Rev. D 72, 123521 (2005) arXiv:hep-ph/0502177.

[15] K. Freese, J. T. Liu and D. Spolyar, "Chain inflation via rapid tunneling in the landscape," arXiv:hep-th/0612056.

[16] B. Feldstein and B. Tweedie, "Density perturbations in chain inflation," arXiv:hep-ph/0611286.

[17] D. N. Spergel et al., "Wilkinson Microwave Anisotropy Probe (WMAP) three year results: Implications for cosmology," arXiv:astro-ph/0603449.

[18] S. R. Coleman, "The Fate Of The False Vacuum. 1. Semiclassical Theory," Phys. Rev. D 15, 2929 (1977) [Erratum-ibid. D 16, 1248 (1977)].

[19] C. G. Callan and S. R. Coleman, "The Fate Of The False Vacuum. 2. First Quantum Corrections," Phys. Rev. D 16, 1762 (1977).

[20] A. H. Guth and E. J. Weinberg, "Could The Universe Have Recovered From A Slow First Order Phase Transition?," Nucl. Phys. B 212, 321 (1983).

[21] M. S. Turner, E. J. Weinberg and L. M. Widrow, "Bubble nucleation in first order inflation and other cosmological phase transitions," Phys. Rev. D 46, 2384 (1992). 